Voix et Images

\title{
III.2 Politiques éditoriales publiées hors BJ/NBJ et inédites
}

Volume 10, numéro 2, hiver 1985

La barre du jour / La nouvelle barre du jour

URI : https://id.erudit.org/iderudit/013872ar

DOI : https://doi.org/10.7202/013872ar

Aller au sommaire du numéro

Éditeur(s)

Université du Québec à Montréal

ISSN

0318-9201 (imprimé)

1705-933X (numérique)

Découvrir la revue

Citer cet article

(1985). III.2 Politiques éditoriales publiées hors BJ/NBJ et inédites. Voix et

Images, 10(2), 41-66. https://doi.org/10.7202/013872ar d'utilisation que vous pouvez consulter en ligne.

https://apropos.erudit.org/fr/usagers/politique-dutilisation/ 


\section{III.2 Politiques éditoriales publiées hors BJ/NBJ et inédites}

\section{Note préliminaire}

Les «archives de la $B J$ », pour ce qui concerne la période qui va des débuts à 1975 environ, ont à peu près complètement disparu dans une inondation, due à la crue printanière de la rivière-des-Mille-Iles, du garage attenant à la résidence de Roger Soublière et Nicole Brossard où elles étaient entreposées. Restent, bien sûr, divers documents en dépôt chez l'un ou l'autre des membres des équipes qui l'ont animée.

Quant aux «archives de la NBJ», elles existent, sont accessibles et ont été consultées avec profit. Nous remercions ici Michel Gay et Lise Guèvremont.

\section{Entrevues/BJ (1965-1978)}

Quatre étudiants de la Faculté des Arts (de l'Université de Montréal), Nicole Brossard, Marcel Saint-Pierre, Roger Soublière et Jan Stafford ont mis, après deux ans de travail, leurs projets à exécution en publiant la revue littéraire La Barre du Jour (...).

Initialement, l'équipe de La Barre du Jour avait l'intention de ne publier que les textes des étudiants de l'Université. Puis elle ne s'attacha qu'à la valeur littéraire en soi, et ses pages furent mises à la portée de tous. (...)

Le but premier de La Barre du Jour est de dépanner et d'encourager les jeunes écrivains. «La poésie est en santé au Québec», remarque l'équipe. (...)

La revue en soi ne prône aucune idéologie, n'adopte aucune orientation quelconque. L'on compte uniquement sur la valeur littéraire. Ayant cependant interrogé l'équipe à ce sujet, les quatre se sont prononcés en faveur de l'indépendance et du socialisme.

Sylvie Roche

«Ả propos de 'La Barre du Jour'» Le Quartier Latin, 4 mars 1965. 
«Nous avons fondé la Barre du Jour dans un but bien précis: non pas de publier n'importe quel écrit de qualité, mais de diriger les efforts d'une équipe en vue de définir une politique littéraire. Un peu comme ces revues européennes connues pour leur politique; sans tomber, évidemment, dans les excès de Tel quel!» Avec une certaine amertume, (Roger Soublière) reconnaît que les scissions et les polémiques, si fréquentes pendant les six ans de la Barre, démontrent la quasi-impossibilité de former une équipe vraiment unie.

«(...) Nous ferons dorénavant une Barre du Jour sans équipe. Tous les auteurs qui ont déjà collaboré avec nous, mais qui n'entraient pas dans ce concept d'équipe, pourront nous soumettre des textes en toute liberté. Nous demanderons simplement que les manuscrits soient centrés sur un thème donné. Et ce thème se rattachera forcément à la recherche littéraire, puisque nous voulons garder au moins cette orientation-là.»

Le débat d'idées, le concept d'équipe, de politique littéraire et de «recherche» sont des concepts chers à Roger Soublière, et tout au long de l'entrevue, il les défendra avec énergie: «les autres revues sont là pour simplement transmettre des textes bien écrits. Ce qui nous intéresse c'est découvrir du neuf en littérature, et cela, on le fait moyennant l'union au niveau d'une théorie. Autrement, à quoi bon une revue, aussi bien se faire éditeur.»

Robert Guy Scully «Nos trois revues de création littéraire... en revue» Le Devoir, 6 novembre 1971.

o

«La Barre du Jour, dit Roger Soublière, c'est essentiellement un groupe de personnes qui tentent de susciter des documents à partir de thèmes élaborés en discussion. Après quoi on fait appel à des écrivains susceptibles d'être intéressés par le champ d'études défini au préalable.

«Une des préoccupations de toujours de la Barre du Jour, poursuit-il, c'est une littérature qui, on l'espère, sort des sentiers battus. Ça demeure une revue de littérature québécoise. Je ne vois pas comment on peut séparer les deux. La littérature s'insère dans une société en évolution et marque cette société-là. La littérature témoigne des transformations et des nouvelles structures d'une société. C'est faire fausse route que d'imaginer qu'existe une littérature qui fasse abstraction du politico-social. C'est particulièrement évident à la lecture du numéro Parti pris (...).»

(...) «On pensait que ça serait amusant de lancer une revue, puis (en 1965 ) on la fonda tout simplement. Nous voulions affirmer une littérature québécoise face à la littérature française. De là la chronique des Inédits qui tente de mettre en lumière notre patrimoine culturel.» 
«Sur un plan bien terre à terre, conclut Roger Soublière, on s'est aperçu que ce qui intéressait le lecteur, c'était les études beaucoup plus que les textes de création. Ça s'explique facilement si on considère que la revue, en ce qui concerne la création, est un banc d'essai.»

Michel Beaulieu

«Malgré tout, il y a des revues littéraires qui survivent»

Perspectives, 21 octobre 1972.

o

«À l'origine de la revue, en 1967 (sic: 1965), nous avions choisi la «Barre» pour signifier qu'une nouvelle littérature commençait qui n'était pas la littérature canadienne-française. La Barre du Jour entendait publier de jeunes auteurs et évacuer de nos textes le thème du pays. Nous voulions aussi renouer avec un passé littéraire jusqu'alors pratiquement inconnu. Une rubrique «Inédits» permet (...).

Marc Kravetz

"Nicole Brossard: une revue des livres, un journal"

Le Magazine littéraire, Paris, mars 1978

o

\section{Exploitation de l'imagination québécoise}

(extraits d'un texte signé collectivement)

(...) une revue comme la nôtre se doit d'ouvrir le plus possible ses pages à ce qu'on ose encore appeler «l'avant-garde» ou la recherche littétaire. De quelque forme qu'elle soit, celle-ci est la seule capable un jour de faire changer ou évoluer notre littérature (un peu trop, dans l'ensemble, traditionnelle).

Toute revue littéraire, dite "spécialisée», se doit, du moins ici, au Québece, d'être «d'avant-garde», si elle veut vraiment faire quelque chose et non répéter «l'ancienne». Toute revue d'avant-garde a un contenu révolutionnaire latent, car en créant une littérature d'avant-garde, celle-ci démolit les anciennes structures; et en un sens est un engagement politique. Une révolution ne peut être que globale. Ni nécessairement telle ou telle (i.e. politique, économique, religieuse, culturelle, etc.), cette révolution ne peut que toutes les grouper. Et cette littérature, ou cet engagement, qui n'est que pleine conscience, ne peut que servir, un jour, de quelque façon, la révolution. La littérature est révolutionnaire car elle permet de faire et de changer la mentalité.

\section{(...)}

Toute recherche formelle se fait strictement au niveau des mots; peu importe les idées ou quoi que ce soit. La recherche formelle, c'est la convention poussée à bout. C'est la convention qu'il faut briser, puis changer. Car 
toute forme est convention (un jour ou l'autre) et doit être mise en question si on veut qu'elle soit communicable.

C'est surtout avec la chronique des Inédits que $\mathrm{La}$ Barre $\mathrm{du}$ Jour a pris position concrète et active face à la tradition. La revue est sans parti-pris de jugements de valeur devant les œuvres traditionnelles. L'objectif premier de cette chronique est sans doute de participer à la recherche et d'inciter surtout le public de la revue à la lecture des ouvres du passé, et ainsi, accorder à ces oeuvres une première génération de lecture véritable; cette tradition de lecture qui est une condition majeure de survie d'une littérature.

Par une chronique future, (EXPLORATIONS) la revue entend montrer les grandes images qui dominent notre littérature passée: faire un éventail qui se voudra chaque fois très restreint, mais qui, nous l'espérons, pourra déceler à la fois des valeurs littéraires et imaginaires. Pour valoriser la tradition, il ne suffit pas de remonter à des sources psychologiques ou sociologiques, il faut faire l'inventaire de notre imaginaire. Nous ne nions pas que notre passé littéraire soit contestable et contesté encore de nos jours; surtout en tant que valeur littéraire réelle. Mais notre revue reconnaît d'abord, l'urgence de l'établir de façon diachronique, de sorte que les De LaHayes, les Loranger, les Routhier, les Piché et autres, aient le droit à une première génération de lecture. Ici, nous devons citer l'excellent travail que font les centres de recherche en littérature québécoise. Car, il est entendu qu'une revue ne peut promouvoir que partiellement une telle entreprise.

L'homme ruine inlassablement le monde, et la recherche formelle, elle aussi, ruine la convention du monde. En ce sens, elle est révolutionnaire. C'est donc dire qu'une revue littéraire n'a rien à perdre avec l'avant-garde et la recherche. Au contraire, elle a tout à perdre avec la convention. Il faut ruiner pour mieux refaire. Et toute règle du meilleur-dire est sans merci.

En ce sens, ce qui compte, pour LA BARRE DU JOUR, c'est la' littérature qui se fait, mais aussi, et peut-être surtout, celle qui se fera; c'està-dire la recherche (actuelle). Nous sommes entièrement pour une littérature nouvelle; littérature qui se doit d'être (un jour prochain) et d'être de recherche (pour le moment).

La littérature pousse là où elle peut, mais elle pousse toujours, à la fois, contre quelque chose qui est et pour ce qui n'est pas (encore). Il est donc temps que nos écrivains et nos poètes lancent réellement leurs cailloux. L'eau-de-rose, c'est fini. Les petites fleurs aussi. Il ne nous reste plus que les mots qui cognent dur. Les mots à faire. Nôtre...Nos...Leurs...

La Barre du Jour Le Quartier Latin, 16 février 1967. 
(autres extraits, signés individuellement)

Il ne s'agit pas tellement de faire éclore de nouvelles thématiques et de nouveaux mythes comme de chiffrer leur comportement dans un espace qui s'agrandit, se multiplie ou se rétrécit selon l'importance que nous lui donnons.

Toute forme de langage présuppose un choix: la communication ou l'expression. Pour moi, il ne fait aucun doute que c'est l'expression qui est toute désignée pour remplir un rôle adéquat dans le domaine de l'écriture.

Le mot, unité palpable, s'apprivoise dans l'éclatement, de la rupture. Il s'agit de fabriquer le mot passe-partout qui ne renvoie plus uniquement à des notions affectives et intellectuelles, mais qui surtout crée ses propres variantes: le mot-conscience par opposition au mot-convention. Il s'agit de prendre en main une logique qui est distorsion et nuance, d'où extension du système sémantique.

J'imagine très bien une poésie où le mot en devenir chercherait à établir une juste relation avec d'autres mots en constante mutation.

Nicole Brossard

La littérature est écriture, et écrire n'est pas parler. Elle est telle et ne doit être rien d'autre. Le temps des chansons est fini. On les connaît toutes. La littérature, c'est des mots et encore des mots... toujours changeants.

Les mots n'ont pas de sens. Ils ne sont qu'illusions et par le fait même fermés à la véritable "communication». La littérature et plus précisément les mots, sont incompréhensibles. Il faut donc que la littérature cesse d'être ce qu'elle n'est pas, c'est-à-dire ce qu'elle est encore, pour se faire autre. Nouvelle. Il lui faut donc tendre irrémédiablement vers un nouvel intelligible.

Une nouvelle compréhension qui se ferait à partir des mots - c'est-àdire de et par eux - implique que la pensée vient des mots et qu'ils sont les seuls compréhensibles possibles. Devenus inutilisables, il nous faut donc les redéfinir inlassablement. Le mot est présence de l'absence. Il est ce qu'il est, et dès qu'un mot est écrit, il ne peut être autre qu'être lui-même. En aucune façon, le mot ne peut se passer de lui-même. Et c'est pourquoi on peut dire que la littérature est à faire.

La littérature, considérée comme en tant qu'exclusivement écriture, n'a jamais existé au fond. Elle n'est pas à dire, mais à voir. Elle doit se faire à l'oeil et aux mots. Rien d'autre. Et c'est tout. 
Le mot, et par suite toute l'écriture, a une double possibilité inhérente à lui-même: d'abord, le mot a une vitalité qui ne joue pas en pure perte dans l'écriture. Mais seul, en tant qu'unité, il se définit comme opacité. Il est en aphasie. Le mot est moindre par rapport au mot déterminé. Par exemple, le mot table est moindre par rapport à table tam-tam. Les mots conjoints s'interpénètrent l'un l'autre et prennent une seule signification qui est spécifiée par les conjoints. Les mots peuvent se multiplier par les joints. Ils portent en eux des charges explosives parce qu'ils sont poly-formes, poly-sons, polysignifiants. Dans l'union, les mots sont à la Nième puissance, ils sont la possibilité de survolter l'écriture.

Par conséquent, les mots doivent être soumis à une détermination précise, c'est dire que l'écriture doit se faire au pied du mot. Le mot doit retrouver une unicité certaine, une pureté initiale, mais est-ce possible dans notre contexte culturel? Le mot doit être précisé, ceinturé, pris au pied et à la lettre. C'est ce que nous pourrions nommer: ce qu'il nous reste à faire.

Toute écriture, dans ce contexte, est une entreprise linguistique qui vise à préciser, toujours davantage, chaque mot. Et peu importe la façon...

En ce sens, écrire n'offre qu'une seule possibilité: transformer.

France Théoret

Le Quartier Latin, 16 février 1967.

\section{Texte présenté à l'équipe de la BJ, en l'année pré-révolutionnaire - 2, 1969-1970}

la BARRE DU JOUR est une revue exclusivement québécoise parce qu'elle est le lieu de rencontre d'auteurs québécois

parce qu'elle s'inscrit dans un contexte québécois, lequel est le résultat non seulement des tendances typiquement québécoises (dépossession, tradition littéraire des thèmes et des formes, re-possession, engagement politique) mais aussi des tendances environnantes (européennes, de moins en moins; américaines, de plus en plus)

parce qu'elle publie, dans ce contexte, des œuvres québécoises

parce qu'elle est non pas le reflet de pensées diverses (revue Liberté) mais son produit; entendons par là que

la BARRE DU JOUR n'est pas qu'une revue du passé

«bien qu'elle se voit inscrite par la force des choses dans un contexte géographique, natonal et culturel, elle a le devoir de le faire évoluer ou changer par les moyens qui lui sont propres, en soutenant par ses publications un esprit et une pensée qui, loin d'être traditionnels ou de suivre les modes, s'inscrivent néanmoins dans un présent qu'elles sont susceptibles, 
par la conscience et la connaissance qu'elles ont de ces réalités, non seulement d'égaler ou de rivaliser, mais de surpasser par la création d'une littérature et d'une critique originale, spécifique à un groupe, et dont les exigences premières seraient...» 1

de présenter sous forme de textes principalement, ou en s'aidant des techniques modernes d'impression et d'expression sur papier, un ensemble d'œuvres faisant état des connaissances établies et de la conscience acquise face à la littérature; car avant tout

\section{la BARRE DU JOUR doit être une revue de création}

qui permette de préférence une recherche de groupe dans la mesure où le groupe est d'accord sur cette recherche

qui permette une recherche individuelle de tous ceux qui en font partie, espérant, en raison de l'équipe existante, que ces recherches individuelles se situent de plus en plus près les unes des autres

qui permette l'expérience, si elle fait état d'un progrès vers une plus grande expressibilité du langage

qui permette également l'expérience qui saura rendre la littérature bourgeoise (notions des TEXTES intouchables, de texte-vérité, de texte «objectif») à son accessibilité première

ainsi

la BARRE DU JOUR sera une revue de recherche

au sens où son équipe prendra pour acquises les expériences littéraires qui nous précèdent et ne les répètera que s'il n'y a que ce moyen pour les dépasser

au sens où toute expérience nouvelle sera d'abord considérée comme telle et non pas dans ses aspects de déjà-vu

au sens où toute oeuvre devra être le sujet d'une véritable discussion où l'auteur aura à justifier la nouveauté de son texte

ainsi, également

la BARRE DU JOUR sera une revue de polémique

et, en ce sens, elle doit accorder autant d'importance à la critique qu'à la création, celle-ci étant seule à pouvoir permettre l'élaboration claire d'une stratégie littéraire susceptible de déborder sur les plans culturel, social et politique

«Tout cela présuppose donc le désir de parvenir à formuler, un jour, individuellement ou, de préférence, en groupe, un art écrit nouveau, original et spécifique appuyé sur une réflexion, une critique et une pensée cohérente et rigoureuse, qui, contrairement à ce qui s'est toujours fait, se

(1) extrait d'un manifeste-document-critique précédent (M. St-P.). 
manifesterait ouvertement tant au niveau de ses textes de création qu'au niveau de ses critiques.

Cet art et cette pensée, nous croyons les entrevoir en posant le problème de la littérature dans la littérature québécoise...» 2

1. la littérature québécoise a désormais ses origines; il en viendrait à personne l'envie de re-soulever le problème de son existence.

2. ses origines sont multiples; distinguons-en trois (l'Europe, le Québec et l'Amérique); d'abord d'inspiration européenne (Nelligan y compris) à cause de l'instruction française reçue par nos écrivains, puis se libérant de ces influences pour trouver ses propres tendances, notre littérature s'est lancée dans l'expression de son incertitude et du déchirement des hommes d'ici.

3. cette littérature (de St-Denys Garneau à Michel Tremblay, en passant par Renaud, Benoît, Godbout et Ducharme, et, vaguement, par Blais, Martin) s'est appropriée les termes de la difficulté d'être, éloignant la mentalité française à l'intérieur même de sa langue, jusqu'à traduire désormais ses réalités dans le langage de la majorité et selon les moyens plus populaires, par exemple, du théâtre que de la poésie.

4. on peut à juste titre reprocher à cette littérature les techniques mêmes de son appropriation qui tendent à ne faire qu'exprimer cette dépossession sans l'assumer réellement, ni la dépasser (comme le fait Miron, par exemple), n'aboutissant qu'à un certain narcissisme de déchéance ainsi qu'à l'accroissement de cette dépossession dans la mesure où elle ne devient pas plus communicable du simple fait qu'elle essaie de parler comme tout le monde, alors qu'elle utilise un procédé. (la littérature, l'édition) qui n'en a pas les moyens...

5. d'autre part, on peut dire qu'historiquement, la littérature que nous appelons «joualisante» a eu son importance et continue d être le jalon d'une plus grande possession d'elle-même et de notre devenir.

6. de plus, par ses implications politiques (et c'est ici la deuxième tendance), cette littérature a le mérite d'avoir fait état d'une opposition revalorisante, à une époque où, en dehors d'elle-même, peu de secteurs d'activités sociales étaient en mesure de le faire.

7. mais, depuis, une littérature spécifiquement révolutionnaire a vu le jour et des Québécois comme Vallières et Bergeron ont, de même que des publications de plus en plus nombreuses (CSN et autres organismes politiques), présenté logiquement les raisons de notre volonté de repossession (nous vaincrons).

8. sans doute notre littérature ne s'en trouve pas nécessairement libérée de la lutte qui concerne tout le Québec; au contraire doit-elle en tenir

(2) id. cette première partie du présent texte, vous l'aurez remarqué, reprend les divisions et le déroulement du texte cité et connu (que vous trouverez peut-être en appendice). 
compte et en faire état à l'intérieur de l'ensemble des différentes tendances dont elle vit.

9. une troisième tendance est le contexte culturel américain de notre écriture; d'existence frâiche, l'influence de la culture américaine (nouvelles techniques d'impression, poster, pop music, pot, sex, and t.v.) (see you later) sur notre littérature est en passe de devenir l'une des plus fortes en raison de nos institutions qui en sont de plus en plus imbues, de notre culture qui y découvre de nouvelles façons de penser et de VIVRE, de notre littérature même, au niveau non seulement du renouvellement des techniques d'écriture ou de publication, mais aussi dans la remise en question des valeurs qui la sous-tendent et qu'elle véhicule.

10. c'est ainsi que, du rejet des croyances religieuses ou commonwealthiques, aux découvertes des nécessités politiques et à l'éclatement de certaines barrières culturelles, notre littérature en arrive à ne plus faire état que des réalités québécoises mais également des nouvelles perceptions de ces réalités.

11. parler de l'Amérique n'est qu'une façon de nommer l'ensemble des possibilités nouvelles offertes par des groupes de recherches comme celui des formalistes, par des individus comme Marcuse, McLuhan, Asimov, Krishnamurti, Wolfe, ou des groupes comme les Beatles, les Who, les Rolling Stones, les Pink Floyd, les Jefferson Airplane...

12. ces possibilités nouvelles nous offrent des critères nouveaux qui situent la littérature à l'intérieur d'un processus de conscience plus complexe face à la littérature (non seulement dans son mode, mais dans son existence même) et qui tendent à n'y voir qu'un procédé, ou situable uniquement par rapport à lui-mème, ou destiné à être réformé vers une structuration plus grande du langage, vers une déstructuration inverse ou encore vers son inclusion dans d'autres procédés, quand ils ne vont pas jusqu'à sacrifier l'écrit au profit d'une civilisation dite de l'«image».

13. notre littérature subit, qu'on le veuille ou non, les contrecoups de cette nouvelle culture dont font état des ecrivains comme Chamberland, Yaugud Duguay, Vanier, Bertrand, auxquels viennent se joindre ceux qui, ancrant plus loin leurs origines, relancent les poèmes-affiches ou la poésie en cubes (Tanguay).

14. on peut dire qu'il en résulte des ouvres nouvelles, ambiguès encore, mais qui n'ont peut-être que pour seul défaut d'oublier parfois le contexte (désormais plus que Québécois) dans lequel elles s'inscrivent pour se prétendre seules nouvelles.

15. d'autre part, on peut reprocher (comme on peut le faire n'importe quand) aux littératures issues des principes complexes de cette «nouvelle culture» de tomber dans la facilité; notons cependant qu'il est tout aussi facile de faire un poème "acide» qu'un texte d'allure «formaliste». 
16. ainsi posé, le problème de la littérature peut se ramenèr efficacement à l'ambiguîté de la situation du MOI de l'auteur dans son langage, de la place de ce langage dans l'ensemble des langages, du langage dans le MOI de la société qui l'entoure, et de cette société telle qu'assumée par l'œuvre.

17. aussi en arrivons-nous à quelques questions essentielles concernant la littérature en devenir que nous désirons produire.

les questions

a. la présence de l'auteur dans son ouvre (emploi du JE, thèmes d'allure romantique et personnaliste ou psychologique, texte «biographique», etc...) est-elle un critère de rejet d'une oeuvre?

cf. «Cette littérature ou son «style» nous croyons...» de M. St-P.

b. la linéarité graphique d'un texte, la seule utilisation des mots (ou d'une langue spécifique) et la présentation traditionnelle d'une œuvre, sont-ils des critères de rejet d'une ouvre?

c. l'accessibilité (que le texte puisse être lu, compris, utilisé) d'un texte est-il un critère de rejet de ce texte?

d. la référence à une école littéraire, à un style ou à un ensemble d'œuvres déjà écrites sont-ils des critères de rejet d'un texte?

e. l'engagement politique (phénomènes de langue ou de situation) d'un texte est-il un critère de rejet de ce texte?

appendice

\section{LA BARRE DU JOUR}

La BARRE DU JOUR est une revue littéraire exclusivement québécoise,

non pas qu'elle veuille confiner la littérature à une nation spécifique, et cela, au détriment des autres littératures, mais parce qu'elle sent le besoin et voit l'obligation dans laquelle elle se trouve de lui accorder, par les moyens qui lui sont propres, l'importance et la valeur que trop souvent on lui refuse, en se donnant pour devoir d'insister sur son passé en publiant, d'une part, des textes inédits d'auteurs décédés susceptibles, en quelque sorte, de l'enrichir et, d'autre part, en encourageant l'analyse de ce passé littéraire par la publication d'études, d'essais ou de théories susceptibles, parce qu'elles supposent la formulation d'une critique sévère de ses oeuvres, ses institutions, ses tendances et son histoire, d'en renouveler la lecture comme la critique. Mais 
La BARRE DU JOUR n'est pas qu'une revue du passé car,

bien qu'elle se voit inscrite par la force des choses dans un contexte géographique, national et culturel, elle a le devoir de le faire évoluer ou changer par les moyens qui lui sont propres, en soutenant par ses publications un esprit et une pensée qui, loin d'être traditionnels ou de suivre les modes, s'inscrivent néanmoins dans un présent qu'elles sont susceptibles, par la conscience et la connaissance qu'elles ont de ces réalités, non seulement d'égaler ou de rivaliser, mais de surpasser par la création d'une littérature et d'une critique originale, spécifique à un groupe, et dont les exigences premières seraient, par exemple, de ne pas ..., mais au contraire de...

La BARRE DU JOUR, c'est aussi une revue de création qui,

jusqu'à présent, s'est contentée de promouvoir la littérature en publiant des textes de création d'auteurs connus ou inconnus offrant à la fois la possibilité, soit d'une certaine continuité, soit d'un certain renouvellement ou, encore, d'un certain dépassement de cette tradition littéraire, n'entend plus se contenter désormais de textes n'offrant qu'une agréable lecture, mais prévoit, malgré une plus grande diversité, non pas forcément raffermir ses exigences, mais maintenir un certain "niveau» des textes, trouver un meilleur «consensus» et des critères mieux définis que ceux d'avant-garde, de formalisme, etc...

La BARRE DU JOUR c'est surtout une revue de recherche,

et en cela, elle se doit de susciter en ses cadres, non seulement une autocritique fermée, mais la formation d'une «théorie» littéraire de groupe ayant une politique définie et pouvant, sur plusieurs plans d'action, servir de cheval de bataille. Cette revue, on le devine, n'a pas pour rôle principal de publier ou d'éditer des textes, mais surtout d'articuler une pensée littéraire et un esprit de recherche littéraire, critique et théorique.

La BARRE DU JOUR sera donc aussi une revue de polémique,

et en ce sens elle se doit d'accorder plus d'importance à la critique qu'à la création car celle-ci est la seule capable de susciter un tel esprit et d'élaborer une stratégie littéraire susceptible de déborder sur les plans culturel, social, politique et historique. Tout cela présuppose donc le désir de parvenir à formuler, un jour, individuellement ou, de préférence, en groupe, un art écrit nouveau, original et spécifique appuyé sur une réflexion, une critique et une pensée cohérente et rigoureuse qui, contrairement à ce qui s'est toujours fait, se manifesterait vertement tant au niveau de ses textes de création qu'au niveau de ses critiques.

Cet art et cette pensée, nous croyons les entrevoir en posant le problème de la littérature dans la littérature québécoise...

Cette littérature ou son "style», nous croyons en avoir reconnu la spécificité dans sa seule écriture et prétendons, la ramenant à elle-même, trouver là seulement son avenir ou, encore, l'avenir de son prochain dépassement. 
Cet art, cette pensée, ce style et cette théorie, nous croyons qu'ils se manifesteront en tant que seule recherche d'une spécificité de l'écriture sous le couvert d'une mise en respect de plus en plus consciente et efficace de l'expressivité d'un moi (celui de l'auteur), depuis toujours en proie aux pires fantaisies, aux pires facilités, à la pure inconscience et, par le fait même, à la tentation de la littérature.

Nous croyons donc que la littérature, ou du moins son idée, doit mourir au profit de la pratique de son écriture et que seule sa pensée peut y survivre.

Marcel Saint-Pierre (inédit)

$\mathbf{0}$

\title{
Présentation de la BJ
}

La BJ est une revue consacrée exclusivement aux lettres québécoises. C'est une revue, dirigée par de jeunes écrivains, qui publie principalement des textes de création, mais on y rencontrera occasionnellement des études critiques sur des auteurs du Québec.

On y trouve, à chaque numéro, une chronique, intitulée Les Inédits, qui a pour but de mieux faire connaître notre passé littéraire par la publication de textes - accompagnés d'une présentation de l'oeuvre demeurés inédits d'auteurs décédés.

\author{
Direction: Nicole Brossard, \\ Marcel Saint-Pierre, \\ Roger Soublière \\ dans la brochure publicitaire intitulée \\ Revues littéraires du Québec, présentant \\ «les trois revues littéraires importantes" suivantes: \\ Etudes françaises, Liberté et la BJ, 1972
}

o

\section{Publicité/BJ (1965 et 1976)}

Vous aimez la barre du jour

Abonnez-vous!

C'est un service que vous vous rendez! 
LA BARRE DU JOUR a été fondée en février 1965. Elle publie des textes de fiction, des études sur les cuvres littéraires québécoises, ainsi que des analyses sur les phénomènes de production qui mettent en cause certains mécanismes de création. LA BARRE DU JOUR veut renouveler les habitudes de lecture et susciter une réflexion sur l'objet même de la littérature. C'est une revue de poésie vivante.

0

\section{Lettres de refus/NBJ (1978-1981)}

équipe Collette-Gay-Brossard-Bouchard

(...) ce type de discours: que vous le disiez savant ou calé, je continue de croire qu'il s'agit d'un discours universitaire (d'universitaire), et, vous l'avouez vous-même, d'une "querelle de chapelle»; la NBJ a choisi de travailler en dehors de ce procès et de se consacrer à la création, à la stimulation de celle-ci ainsi qu'à la (mise en) circulation de la pensée (créatrice aussi). Cela ne signifie pas que certaines recherches ne puissent pas nous intéresser; (...)

Vous comprenez alors que ce n'est pas votre article en soi que nous refusons, mais bien la surface où il s'inscrit (recto ET verso).

0

(...) nos critères de sélection font que nous publions surtout des textes de la marginalité formelle; ce qui n'est pas du tout le cas pour les poèmes que vous nous avez envoyés.

0

(...) en ce qui concerne nos critères, celui qui s'applique le plus souvent est celui de l'unanimité en faveur de la publication.

Et bien sûr, un texte refusé n'est nullement «une fermeture hermétique» aux portes de la Nouvelle Barre du Jour.

0

Je comprends que vous préféreriez voir paraître un ensemble et c'est ce que nous-mêmes souhaitons généralement. Mais nous considérons aussi la revue comme un lieu de travail et la parution de fragments s'inscrit facilement dans cette perspective. 
(...) la nbj tente de témoigner d'écritures plus «modernes», avec tous les risques qu'une telle entreprise comporte, y compris bien sûr le risque d'errer plus souvent qu'à notre tour. Ainsi, croyons-nous que votre travail manque d'audace, que vos textes ont de la difficulté à sortir des sentiers battus. Votre style y gagnerait peut-être à être un peu secoué.

o

Ne pas craindre de couper quitte à ne produire que de très courts textes. Vaut mieux deux pages et demie de prose étonnante que sept pages qui captent plus ou moins l'attention du lecteur. Le titre serait peut-être à reconsidérer; ne reproduit-il pas quelques manies d'écriture.

o

Nous croyons que l'écriture est plurielle et qu'il faut travailler à en dévoiler les multiples facettes ainsi qu'à en créer de nouvelles. Votre travail n'est pas tout à fait dépourvu d'intérêt. Il nous semble cependant que vous auriez avantage à «murir» vos textes, apprendre à les élaguer; cela demande du temps et beaucoup d'investissement, beaucoup d'énergie. Écrire énormément pour obtenir un peu de «bon texte».

o

Je ne sais trop comment interpréter le travail de (X). Si c'est un clin d'oeil adressé à la NBJ, nous l'en remercions et le prions de récidiver en produisant quelque chose, mettons, de moins «évident».

o

La NBJ préfère, de son côté, des textes moins ancrés dans la tradition.

0

Nous ne nous opposons pas à la recherche, loin de là, mais nous essayons de publier des textes qui offrent aussi des «trouvailles». De ce côté, votre travail nous a laissés sur notre faim.

o

Nous croyons en effet qu'une revue comme Estuaire serait mieux désignée pour les accueillir. Il en va, si vous voulez, d'un certain type d'écriture que nous désirons privilégier. Cela n'enléve rien à la qualité de votre travail. Celui-ci contient d'ailleurs un certain nombre d'éléments qui tombent sous la coupe de ce que l'on appelle l'avant-garde. Mais l'ensemble relève, selon nous, d'un autre ordre de poésie. Et de préoccupation(s). 
Nous ne pouvons malheureusement pas non plus répondre à la question que vous soulevez dans votre lettre au sujet des critères de sélection (et de rejet). La littérature, selon le point de vue de la NBJ, se définit «à l'usage». C'est donc de numéro en numéro que nous essayons d'affirmer les «différences» littéraires. Nous investissons d'emblée, il va sans dire, du côté de la modernité.

0

Il nous semble qu'il y a dans vos travaux des relents d'écriture automatique qui ne sont pas des plus productifs (danger de toute technique d'ailleurs).

o

Bref donc: vous donnez l'impression d'avoir lu la poésie québécoise et de l'avoir suivie, en partie au moins. Cependant, vos textes ne semblent en projeter que le signe. Peu de substance. La question n'est pas, elle ne sera jamais, de «faire» moderne. (...) En outre, une écriture moderne coïncide avec une pensée également moderne. Des propos judaïco-chrétiens reviennent trop souvent sous votre plume.

\section{0}

Comme vous l'avouez vous-même dans votre lettre, ça manque un peu d'expérience. Comment acquérir celle-ci? En lisant, sans doute, en lisant beaucoup. En suivant ce qui se passe en poésie au Québec et ailleurs dans le monde. La poésie n'est pas une idée, même pas l'idée qu'on peut s'en faire ou ne pas s'en faire. La poésie est une pratique.

On a l'impression que vous craignez de laisser aller le poème. Vous semblez vouloir reproduire la phrase. Si c'est la phrase qui vous intéresse, il faudrait peut-être essayer la prose. Il faudrait l'essayer de toute façon. Il faut tout essayer. Il faut tout écrire. L'écriture, je veux dire votre écriture, le poème ou la prose de $(\mathrm{X})$, viendra là-bas, de là-bas, de là où l'on écrit, où l'on lit, sans cesse.

Pour revenir à vos poèmes, il nous semble qu'il y a un peu trop de volonté d'anecdote, un peu trop de morale aussi. Le poème n'est pas un discours, c'est l'anti-discours par excellence. Je ne vous dis pas de ne pas en faire, je vous dis de ne pas vous servir du poème pour en faire. Le poème, c'est du travail, c'est de l'écriture travaillée, c'est du travail écrit.

Continuer - et oublier ces remarques: voilà ce qui importe!

Lettres rédigées, à l'exeption de deux, par Michel Gay (inédit) 


\section{Lettres/NBJ (1978-1979)}

Une politique du découragement

La revue La Nouvelle Barre du Jour vient de recevoir sa subvention du Conseil des Arts du Canada. L'octroi est soumis a certaines remarques et conditions. L'équipe de la revue a réagi dans uné lettre ouverte qui veut démontrer les contradictions qui auraient régi l'octroi de sa subvention par le Conseil des Arts.

Au mois de septembre dernier, avec sa cinquante-huitième livraison, une revue littéraire qui agissait dans le milieu depuis déjà une douzaine d'années et dont la contribution jusque là n'avait pas été des moindres, trouvait la force et, disons-le d'emblée, l'imagination nécessaires à son propre renouvellement. Un bon millier de lecteurs auront reconnu $L a$ Nouvelle Barre du Jour.

Nouvelle, cela veut dire d'abord une nouvelle équipe de direction: Nicole Brossard, Jean Yves Collette et Michel Gay se réunissaient dès l'hiver 76-77 pour penser, organiser et finalement créer une revue littéraire différente. Nouvelle, cela veut dire un nouveau rythme de publication: douze (12) numéros par année au lieu des très classiques et très habituels quatre ou six. Nouvelle, cela veut dire une présentation soignée et vivante: chaque livraison comprend un certain nombre de rubriques parmi les suivantes: Fictions, Histoire d'écrire, Essai, Intervention, Signaux et Commentaires, chacune d'elles apportant à l'écriture un éclairage spécifique, chacune d'elles provoquant à l'écriture. Nouvelle, cela veut dire surtout que la revue entend suivre de près, sinon annoncer, la littérature québécoise dans les voies diverses où elle s'engage.

Tout cela a été soumis à l'attention du Conseil des Arts du Canada et a même fait l'objet de consultations auprès de cet organisme, car la direction de la revue voulait s'assurer au moins d'un appui «moral» avant de lancer la machine. Cet appui, La Nouvelle Barre du Jour croyait l'avoir obtenu et c'est avec confiance qu'elle présentait, l'été dernier, sa demande de subvention. Est-il nécessaire de préciser que les revues littéraires ne sauraient survivre sans une aide quelconque, que cette aide vienne d'un Conseil des Arts ou d'autres organismes semblables. On comprendra notre stupéfaction lorsque le Conseil nous fit savoir, au moment de verser la subvention, que son "comité» trouvait irréaliste notre projet et que nous devrions nous limiter à... huit numéros par année. Un nouveau chiffre était lancé dans la loterie du Conseil et j'imagine que quelques membres du «jury» ont da fêter au vin cette belle trouvaille.

La Nouvelle Barre du Jour, dont le premier numéro venait de paraître, dont le second était déjà sous presse, et dont les suivants accueillaient dès lors bon nombre de textes, La Nouvelle Barre du Jour qui avait prévenu ses abonnés et les agences qui acheminent les abonnements, $n$ 'entendait pas, on le comprendra, se plier à ce jeu de roulette russe. Elle rattaquait au Conseil 
de Arts - des sorts? - pour conclure, verbalement bien sûr, une entente qui semblait satisfaire les deux parties: cette subvention vaudrait pour six numéros, la revue pourrait soumettre une nouvelle demande, cette fois pour douze numéros, fin février.

Et le manège recommence à tourner. Mais cette fois La Nouvelle Barre $d u$ Jour n'est pas qu'un projet; c'est une réalité qui se touche et se lit à travers les six numéros et les six cents pages qu'elle a publiés de septembre à février. Les numéros de mars et avril sont sortis et le numéro de mai est sous presse, comme il se doit, quand nous recevons l'irrecevable:

«Lorsqu'il s'est réuni pour étudier votre dossier notre comité a trouvé votre projet quelque peu audacieux. Votre intention de publier une fois par mois nous a paru inopportune; mieux vaudrait, selon nous, que la revue paraisse tous les deux mois et qu'elle assure ainsi une meilleure qualité intrinsèque.»

La NBJ, un projet audacieux? Il peut sembler audacieux à certains qu'une jeune revue demande à Jacques Brault, à G.-André Vachon, à PaulMarie Lapointe, à Roland Giguère, à Louis-Philippe Hébert ou à Michel Beaulieu de prendre les pages qu'il faut pour penser leur travail d'écrivains et écrire ce travail; audacieux de publier des essais de Paul Chamberland (Mythe ou fantasme), Yolande Villemaire (La grande ourse, configuration du désir et de la peur, schéma de la possession), Laurent Mailhot (Aux frontières de l'essai québécois), Joseph Bonenfant (Masculinarités), Marcelle Brisson (L'écriture réflexive au Québec), Claude Beausoleil (Du texte et du doute), et Normand de Bellefeuille (Le corps mineur ou l'impossible lyrisme); audacieux de publier des commentaires sur des livres dont personne ne parle dans la presse écrite ou électronique; audacieux de permettre aux écrivains et aux lecteurs québécois de connaître un point de repère québécois; audacieux de publier, côte à côte, les écrivains les plus mûrs du Québec et les nouveaux auteurs qui cherchent à être lus, avec raison, parce que leurs textes le méritent; audacieux tout cela? peut-être. Mais inopportun? Il faut ne pas connaître les mécanismes de la littérature, je veux parler d'une littérature vigoureuse, vivante et sans cesse renouvelée, pour ne pas se rendre compte de l'opportunité qu'offre, justement, un périodique comme la NBJ: aux écrivains qui peuvent publier leurs textes sans souffrir les délais de plus en plus longs de l'édition de livres où il n'est pas rare de laisser "vieillir» un manuscrit deux ou trois ans; aux lecteurs qui peuvent suivre les tendances de la littérature ailleurs que dans les pages publicitaires des journaux, ailleurs qu'aux vitrines des librairies où il n'y a de place que pour les best-sellers américains et européens, ailleurs qu'à la télévision où le livre n'existe pas; à l'écriture, enfin, pour qu'elle se développe et donne naissance à des ouvres nouvelles. Inopportun? Nous renvoyons le Conseil des Arts du Canada aux manuels de littérature de l'an 2000. Allez-y voir!

Quant à la «valeur intrinsèque» de La Nouvelle Barre du Jour, il suffit de jeter un coup d'oeil aux sommaires des numéros 58 à 65 pour l'affirmer. La confiance des écrivains de toutes les générations qui y collaborent, le 
chemin que la revue a parcouru dans les différents lieux littéraires du Québec depuis sa fondation (depuis huit mois), les énergies qui ont été investies jusqu'd̀ maintenant et qui continueront de l'être ne constituent que quelques-unes des «garanties» qui accompagnent le moteur.

Le CAC, pour sa part, roule au neutre depuis assez longtemps. Il n'y a, pour s'en convaincre, qu'à rapprocher une certaine brochure intitulée 20 +5 , dans laquelle le CAC souhaite définir ses orientations prochaines, de la situation très actuelle qui nous préoccupe. À travers ces pages, le CAC cherche à provoquer une réflexion qui conduirait au renouvellement de sa politique d'aide à la création; devant la réalité de la NBJ, le CAC veut nous faire faire marche arrière et nous cantonner dans un statu quo où s'enlisent les revues littéraires depuis vingt ans. D'autre part, comme on a pu le lire dans les journaux récemment, quand le CAC fait un «effort d'imagination", c'est pour «inventer» une "semaine nationale du livre» qui ne sera qu'une carosserie chromée pour recouvrir et camoufler la formule usée des salons de livre. Ainsi, le CAC préfère-t-il mettre en place une machinerie «nouvelle» pour fabriquer le même vieux produit et pèse-t-il de toute la lourdeur de sa bureaucratie pour empêcher l'éclosion d'un véritable renouveau.

La Nouvelle Barre du Jour, l'a-t-on deviné, n'a pas l'intention d'abandonner la partie. C'est avec une demi-subvention, au prix toujours des droits d'auteurs, au prix des énergies énormes qu'il faut investir dans une telle entreprise, mais avec la confiance des écrivains et l'appui des abonnés présents et à venir, qu'elle va persister: La Nouvelle Barre du Jour continuera d'offrir aux lecteurs québécois douze (12) numéros par année.

Michel Guay,

La Nouvelle Barre du Jour

Le Devoir, 29 avril 1978.

(...) ce n'est pas à un groupe d'écrivains estriens que nous avons fait appel, mais à des gens dont nous aimions les textes et à qui nous pouvions faire confiance. À la nbj, faut-il le préciser, nous nous intéressons à la littérature et les étroites considérations de territoire tout autant que «le bel exemple» ne nous effleurent pas l'esprit, si ce n'est pour les dénoncer.

Jean Yves Collette

(mai 1979, à l'Association des Auteurs des Cantons de l'Est, à propos du no 77). 


\section{Lettres de refus/NBJ (1981-1983)}

équipe Corriveau-Cotnoir-Guèvremont

(...) l'esprit que nous tentons de maintenir à la NBJ. Il s'agit d'une revue qui tente de publier des textes nouveaux, de ceux qui pourraient renouveler l'écriture et les données littéraires elles-mêmes.

\section{0}

(...) une inadéquation entre, disons, leur forme-contenu et la tendance déjà bien marquée de notre revue.

0

Nous travaillons directement à saisir le littéraire dans toute sa modernité, et en regard de cela vos textes nous ont semblé tenir encore d'une trop grande linéarité.

0

Par contre votre travail de recherche ne saurait nous laisser indifférent puisque notre revue a pour but de justement favoriser l'avancée de la littérature.

o

(...) nous aimerions vraiment vous expliquer «la politique éditoriale» de notre revue.

Mais voilà... comment vous expliquer cette politique sans vous suggérer de lire attentivement notre revue qui nous paraît l'expliquer d'ellemême, manifester par elle-même ce qu'elle compte être, ce qu'elle souhaite mettre de l'avant. Comment redire ici qu'il nous paraît évident qu'elle s'ajuste le plus possible à une modernité vivante, $a$ un travail littéraire qui cherche autant à renouveler la forme que les diverses thématiques usées, anciennes, redites, recousues à d'éventuelles influences (...). Se dire aussi ouvert le plus possible sur les approches diverses de la recherche ne veut pas dire qu'on doit (qu'on peut) tout aimer. Il en va du choix des divers animateurs et animatrices de revue comme de tout le reste, il ne saurait se faire sous des critères d'une absolue objectivité. Il est donc certain que refuser (ici le titre du texte refusé) tient autant à un regard critique sur le texte lui-même qu'à une subjectivité propre aux lecteurs que nous sommes. (...)

Ceci dit, il va de soi qu'on ne saurait convaincre tous les auteurs refusés du bien-fondé de nos décisions. S'il fallait dire ici une chose en ce qui concerne vos textes, c'est qu'ils nous sont apparus d'une très haute qualité littéraire mais qu'il ne nous a pas semblé pertinent de les publier 
puisque la forme même que vous leur avez donnée impliquait qu'ils se tiennent tout au bord d'un certain regard suranné.

Ce jugement ne nous tient certainement pas à l'abri du vôtre, et sans doute trouverez-vous à l'intérieur des pages que nous publions certaines raisons de nous prendre en flagrant délit de contradiction. Qu'à cela ne tienne, puisque ce serait la preuve d'une certaine vitalité.

Nous cherchons à ouvrir la voix de la modernité vers l'avancée toujours recommencée du texte, non pas à redire l'émotion romantique devant la nature. Vous demandez des commentaires... quel autre donner que celuilà même qui ne situe pas aujourd'hui le travail que vous avez entrepris. La beauté seule ne saurait être un gage de renouvellement, encore moins un stimulus efficace au désir d'écrire. Alors que dire, sinon l'étonnement qu'encore maintenant on puisse trouver matière à dire la stricte exaltation du paysage.

o

Nous ne croyons pas faire en sorte que se lise chez nous ce vieux fond romantique et naturaliste qui caractérise vos propres textes.

\section{o}

Nous tenons à te signaler l'intérêt que nous avons eu à lire ce texte (incidemment fort bien écrit) mais nous te dirons aussi que nous n'avons pas vu comment il pouvait vraiment s'accorder avec les préoccupations de la revue. Peut-être est-ce do au lyrisme un peu trop insistant de ce texte ou à sa facture ou encore à ce thème même de la difficulté d'écrire qu'il est tellement difficile de renouveler, mais quelque chose là nous est apparu un peu moins transgressif que tes autres textes.

\section{0}

Comme vous savez sans doute, notre revue ne se préoccupe que de ce qui est actuel.

0

Vous demandez (...) avec une certaine naïveté «un ouvrage qui expliquerait les divers «genres», qui dirait pourquoi l'on «doit» apprécier tel ou tel texten! Que vous dire, alors que nous avons justement l'habitude de renvoyer d'éventuels collaborateurs aux textes eux-mêmes afin qu'ils puissent voir au plus près le lieu de la modernité. Or, vous nous dites avoir lu certains textes déroutants, une certaine anthologie que vous glorifiez. Alors que dire, sinon que Jehanne Benoît n'a pas encore écrit «le bon usage 
de la poésie», que tout lecteur reste bien seul pour apprécier tel ou tel texte, mais que le jugement se forme au fil des lectures, que tout jugement se forme quand une certaine ouverture d'esprit (et sans préjugés malheureux) est conviée comme préalable.

Vous vous êtes sans doute rendu compte que la $n b j$ publiait des textes dont le travail d'écriture cherchait, à tout le moins, à déranger le convenu, à multiplier les sens possibles de lectures, a renouveler une certaine thématique usée. (...)

Effectivement, on peut vraiment douter de la valeur littéraire (conventionnellement perçue) de certains textes publiés par notre revue, mais si vous y regardez de plus près, vous verrez sans doute cette volonté de renouvellement qui seule peut vraiment animer l'écriture, la mener ailleurs, la garder vivante. C'est un peu là ce que nous croyons faire en donnant à lire du texte troublant, $c$ 'est un peu en bousculant les habitudes de lecture que l'écriture trouve à son tour un stimulus au changement.

Lire donc, toujours et encore, ces textes de la modernité. Et dites-vous que vous partez avec une bonne longueur d'avance puisque vous vous y intéressez déjà. Si vos questions restent en définitive sans réponse, sachez que votre seul questionnement est déjà une ouverture, ce qui pourrait éventuellement vous mener à votre propre écriture.

Lettres rédigées, à partir des remarques de l'équipe, par

Hugues Corriveau

(inédit)

o

\section{Demandes de subvention/NBJ (1979-1983)}

\section{Objectifs:}

1. Publier des textes de fiction, des interventions, des essais et des commentaires sur les œuvres littéraires québécoises, ainsi que des analyses sur les phénomènes de production qui mettent en cause certains mécanismes de création.

2. Renouveler les habitudes de lecture et d'écriture par la publication de textes engagés dans la voie de la recherche et tributaires des plus récentes explorations de la pensée.

3. Explorer des sujets qui semblent momentanément faire l'unanimité dans l'ordre de préoccupation des écrivains et susciter une réflexion sur l'objet même de la littérature. 
4. Synchroniser l'écriture et les courants de pensée les plus dynamiques, qui élargissent sans cesse nos champs d'action,-de réflexion et de bienêtre.

Michel Gay (décembre 1979)

0

\section{Objectifs:}

1. Publier non seulement des textes de fiction mais aussi et de façon particulière des essais, des interventions et des commentaires sur le phénomène littéraire comme sur les œuvre québécoises, ainsi que des analyses des phénomènes de production qui mettent en cause les mécanismes de la création (...);

2. susciter une réflexion continue sur l'objet de la littérature actuelle et maintenir la nbj comme lieu de convergence des écritures et des courants de pensée les plus dynamiques;

3. renouveler les habitudes de lecture et d'écriture par la publication de textes engagés dans la voie de la recherche et tributaire des plus récentes explorations textuelles;

4. permettre à des responsables occasionnels de réaliser avec des collaborateurs/trices de leur choix des projets particuliers concernant les problèmes de la modernité afin d'en arriver à la publication de numéros spéciaux (...).

0

\section{Publicité/NBJ (1977-1983)}

(octobre 1977-mars 1978)

Rappelons que la Barre du Jour (...) a toujours été considérée comme la seule revue littéraire d'avant-garde au Québec.

(novembre 1977)

0

Nouvelle, cela veut surtout dire que la revue éclaire, sinon annonce, les voies diverses où s'engage la littérature québécoise.

$(1977-1978)$ 
(...) le lieu où émerge la littérature québécoise; le lieu indispensable pour une littérature vigoureuse et continuellement stimulée.

0

(...) l'indispensable périodique littéraire.

(octobre 1977-mars 1978)

o

Le seul mensuel de création littéraire au Québec.

La NBJ est le reflet le plus complet et le plus varié de la littérature québécoise.

(juin 1978)

$\mathbf{0}$

(...) un périodique littéraire qui veut stimuler l'écriture par la fiction, les commentaires et les interventions des créateurs d'aujourd'hui.

0

Créer un véritable périodique littéraire - la revue publie douze numéros par année entre les mois de septembre et de juin -, stimuler l'écriture par l'écriture et donner à lire des textes de fiction d'abord, mais en ouvrant aussi les pages aux essais, aux commentaires et aux interventions nécessaires, renouveler les habitudes de lecture et de production de la littérature, explorer des sujets qui semblent momentanément faire l'unanimité dans l'ordre de préoccupation des écrivains et susciter une réflexion sur l'objet littéraire, synchroniser l'écriture et les courants de pensée les plus dynamiques, qui élargissent sans cesse nos champs d'action, de réflexion et de bien-être: tout ceci ne constitue que quelques-unes des multiples facettes de La Nouvelle Barre du Jour.

La revue entend reconnaître et faire connaître les écritures les plus vigoureuses, d'où qu'elles viennent et où qu'elles aillent. Cela s'appelle travailler à admettre et à renforcer les «différences littéraires». (...)

(...) la volonté que nous avons d'engager lecteurs et écrivains dans les voies de la recherche, dans l'exploration de la pensée, de la pensée poétique, et, pourquoi pas, car c'est bien de poésie qu'il s'agit, du secouement de cette pensée. 
La première fois qu'une lectrice, qu'un lecteur, ouvrira la revue (disons-lui tout de suite que la NBJ sans en avoir le prix a la forme d'un livre), sans doute sera-t-il un peu étonné que cela puisse s'écrire car il se demandera comment le lire. Avec étonnement. Et plaisir, justement.

Michel Gay (novembre 1978)

0

le mensuel québécois de création littéraire.

(avril-mai 1979)

nbj c'est la nouvelle barre du jour.

Une revue littéraire, format livre: 12 numéros par année, plus de 1000 pages! POESIE - PROSE - ESSAIS - CRITIQUES.

On s'y abonne et on la collectionne.

Numéro spécimen et bulletin d'abonnement sont fournis sur demande; envoyez vos nom et adresse à:

La nouvelle barre du jour,

C.P. 131, Succ. Outremont, Outremont, H2V 4M8

(novembre 1979-mars 1980)

D'abord et avant tout: LE SEUL MENSUEL DE CREATION LITTÉRAIRE AU QUÉBEC.

Mais aussi: un lieu de découverte - un plaisir de lecture renouvelé l'espace privilégié de la nouvelle écriture - une revue ouverte aux nouveaux (11es) auteurs (es) comme à ceux et celles qui font aujourd'hui la littérature du Québec - l'avantage aussi d'une revue qui s'offre comme un livre.

(automne 1981)

\section{o}

Mensuel de création littéraire.

(juin 1982-novembre 1983)

o

L'espace privilégié de la nouvelle écriture.

(octobre-novembre 1983) 


\section{Présentation de la NBJ}

Question - Est-ce qu'il y a une différence d'importance entre $L a$ Barre du Jour et La Nouvelle Barre du Jour? Est-ce qu'il y a eu changement d'orientation au cours des années?

HC - $\quad$ Oh, sans doute, énormément (...). La seule permanence qu'il y a eu, dans le fond de la revue, c'a été de toujours être à l'affut de la nouveauté et de ce qu'on pouvait même appeler à l'époque la modernité comme telle, mais au fil des années.

Question - Vous avez parlé tout à l'heure de modernité. Est-ce qu'on vous fait parfois le reproche de la difficile lisibilité de certains textes?

$\mathrm{HC}-\quad$ Oh, très très fréquemment.

Question - À cause du vocabulaire?

HC - Oui oui, très fréquemment.

Question - Est-ce que vous vous en défendez?

HC - Non, on ne s'en défend pas dans la mesure où, effectivement, ce n'est pas une revue qui est d'un abord très facile. Je pense que la revue s'adresse beaucoup plus à des passionnés de littérature qu'à des spécialistes. Je pense que où c'est enrageant, c'est de recevoir comme critique que cette revue-là s'adresse à des spécialistes. Ce n'est, à mnr. avis, quant à moi, pas vrai. Je pense que ça demande simplement une passion, et qui aime la littérature se rend certainement disponible à une écriture un petit peu plus difficile.

Question - On parlait, au début de la revue, d'un groupe formaliste qui publiait cette revue, qui écrivait dans cette roivic. Esil-ce que, encore, vous vous définissez comme des formalistes sur le plan littéraire?

HC - $\quad$ On s'en défend, on s'en défend, je pense. Il en reste très peu de véritables formalistes. À part André Gervais qui continue très sciemment à travailler la lettre du texte, peut-être pourrait-on dire que Michel Gay, qui était de la direction, faisait aussi du formalisme. Je pense qu'on en fait de moins en moins.

Question - Vous vous ouvrez sur toute expérience que vous considérez valable.

HC - Oui, je crois que depuis que La Nouvelle Barre du Jour (existe), elle est beaucoup plus lisible qu'à une certaine période de son existence. 
Question - Est-ce qu'on accorde plus d'importance à la poésie, ou est-ce que c'est vraiment une revue de poètes?

HC - Je répondrai là-dessus qu'on essaie de tenir compte surtout de la qualité.

- Bien répondu.

Entrevue de Hugues Corriveau par Gilles Archambault, Wilfrid Lemoyne et Yvon Rivard Littérature au pluriel Radio-Canada MF, 5 mars 1982

o

\section{Portrait d'éditeur (extraits)}

(...) De tout cela, ce qu'il faudrait avant tout retenir, c'est la force d'entraînement incroyable qu'un tel dynamisme suscite dans le milieu littéraire et dans le paysage culturel québécois. Ce mensuel donne ainsi lieu à autant de polémiques que de vives réalisations. La réelle importance de sa vitalité ne fait plus aucun doute, et la recherche tient là un lieu essentiel à sa poursuite. (...) Nouvelle écriture donc, nouvelle recherche, nouvelle collaboration, nouvelle voix, en somme toute la Nouvelle Barre du Jour.

Hugues Corriveau Louise Cotnoir Lise Guèvremont Littérature du Québec, Bulletin d'informations publicé par l'UNEQ, 1983. 